\title{
The Severity of Menopausal Symptoms and its Relation to Obesity, Smoking, and Exercise in Middle-Aged Jordanian Women
}

\author{
Ruba M Jaber $^{*}{ }^{(\mathbb{D}}$, Hana H Abu Hassan${ }^{1}$, Malaak F Abuelayyan $^{1}$, Jailan N Yasin' ${ }^{1}$, Heba M Jaber $^{1}$
}

\begin{abstract}
Objectives: The present study aimed at finding the effect of menopausal transition, exercise, smoking, and obesity on menopausal transition. In addition, the impact of menopausal transition on women's quality of life was investigated.

Materials and Methods: This cross-sectional study was conducted at family medicine clinics of Jordan University Hospital (JUH). Totally, 359 women whose age varied from 45 to 65 years old were included, Variables including sociodemographic, smoking exercise and obesity, menopause status and its symptoms, and quality of life were assessed by self-administered and validated questionnaire. Data were analyzed using SPSS, version 16.

Results: The mean age at the menopause was 49.4 years. More than $38 \%$ of the participants practiced no or irregular exercise and slightly more than $92 \%$ had abnormal body mass index (BMI) of $\geq 25 \mathrm{~kg} / \mathrm{m}^{2}$. Besides, women who practiced no or irregular exercise were significantly more likely to experience irritability, be mentally and physically exhausted, report sexual problems, and muscles and joint discomfort $(P$ value of $<0.05)$. In addition, women with an abnormal waist to hip ratio were statistically at an increased risk of experiencing sexual problems.

Conclusions: Based on the results, it can be concluded that normal body weight and regular and effective exercise were the key factors in alleviating menopausal symptoms during the menopausal transition, especially the psychological or mental symptoms and muscle and joint discomfort. Meanwhile, women in the midlife stage are recommended to be counseled about menopausal transition and its effect on mental and physical health. Moreover, they should be encouraged to maintain effective levels of exercise and to optimize their body weight whenever possible.

Keywords: Menopause, Obesity, Smoking, Exercise, and Quality of life
\end{abstract}

\section{Introduction}

Menopause is defined as the loss of ovarian follicular activity that results in permanent cessation of menstruation, and it clinically presents as 12 months of amenorrhea. Perimenopause is an ambiguous term that has been used to describe the period of time until 1 year after the last menstrual cycle was menstrual and endocrine changes take place (1). Menopause occurs naturally in all women who survive to middle age, but the results from previous studies indicate that attitudes and perceptions towards menopause vary among different societies (2).

The experience of menopausal transition is very individualized, it is affected by many factors including biological, physiological, sexual, and socio-relational, which all together affect the woman's coping ability in this stressful phase of her life (3). The menopausal period plays an important role in a woman's reproductive life, and it can cause many physical and mental health problems (4). Obesity is an important public health concern that has associations with the menopausal transition (5).

Obesity is considered a worldwide epidemic, and its frequency has increased in both developed and developing countries $(6,7)$. The findings from a large-scale study that took place in Jordan showed that $59.8 \%$ of the women were obese and that the rate of obesity was higher among the older women (8). A high prevalence of obesity among menopausal women could affect the severity of the menopausal symptoms either directly $(1,9-11)$ or indirectly by augmenting the development of other metabolic syndrome components or cardiovascular diseases (10). There is robust evidence to suggest that health-related factors, which include body weight (which is mainly represented by the body mass index [BMI]), smoking habits and exercise, in addition to sociodemographic and economic factors may influence the prevalence of menopausal symptoms (12-16). Thurston and Joffe (9) reported that increased abdominal obesity was related to the increased risk of hot flashes while Gast et al (10) showed that unfavorable cardiovascular risk profiles were linked to hot flashes and night sweats.

In addition, lifestyle factors such as regular effective exercise and smoking cessation might affect the courses 
of many menopausal symptoms (11,17-19). Exercise, smoking, and obesity play an important role in individuals' perceptions of good health and a good quality of life (20).

Quality of life is defined as the perception of the status in life by the individual, which is based on the cultural and value system where that person lives in. Meanwhile, it accounts for an individual's aims, expectations, standards, and concerns $(21,22)$. Women's quality of life can be impaired by the severity of menopausal symptoms. The results of some studies have proven the beneficial effects of reducing these symptoms by using hormone replacement therapy (HRT) (14). Daly et al found that the presence of menopausal symptoms compromised many women's quality of life (23).

There are limited data in Jordan and other countries in the Eastern Mediterranean region regarding studying the factors such as transition through menopause, the severity of menopausal symptoms, the predictors, and the effects of lifestyle factors on women's menopausal courses. Given that lifestyles and obesity trends have dramatically changed worldwide over the last few decades $(24,25)$, it is necessary to investigate the effect of these factors on the lives of menopausal women.

Accordingly, the present study sought to explore the obesity prevalence and to examine lifestyle factors, for example, smoking and exercise, within a cohort of middle-aged Jordanian women who were at different stages of their menopausal transition. Furthermore, the study attempted to determine whether there were any relationships between the aforementioned factors and the menopausal symptom severity. In addition, the impact of lifestyle factors and the obesity indices on menopausal women's quality of life were explored.

\section{Materials and Methods}

Participants and Setting

The present cross-sectional study was implemented at family medicine clinics of Jordan University Hospital (JUH), which is the largest teaching and academic hospital in Amman. This hospital serves a large number of patients including those who are medically insured through the Ministry of Health, university employees, individuals from many affiliated large institutions and companies, and private patients. The family medicine clinics are walk-in hospital-based clinics that serve patients of all age groups who present with acute, sub-acute, or chronic complaints, and individuals who visit the clinic for health check-ups and preventive services.

A total of 359 women who were aged between 45 to 65 years were included in this study. These women referred to the family medicine clinics between February 2014 and November 2014 for a variety of reasons. They were assessed for inclusion criteria, and they were then requested to complete a self-administered questionnaire. The inclusion criteria were being female, aged between 45 and 65 years, present at the clinics during February to November 2014, and agreed to participate in the study. Women who had undergone hysterectomies or oophorectomies, and those who had been diagnosed with and/or treated for any type of malignancy or other conditions that could affect the occurrence or severity of menopause were excluded from the study.

The women were subdivided into three groups according to the status of their menstrual cycles: (I) the premenopausal group including women who had undergone $\geq 12$ regular menstrual periods during the previous 12 months; (II) the perimenopausal status group that comprised women who had experienced irregular or infrequent menstrual periods over the previous 12 months; and (III) the postmenopausal group that were women whose menstrual cycles had been continuously absent for $>12$ months.

\section{Anthropometric Measurements}

All of the anthropometric measurements were undertaken according to the criteria established World Health Organization (WHO). Trained research assistants measured physical characteristics of the participants such as their waist and hip circumferences, weight, and height. All of the measurements were conducted using wellcalibrated equipment that included an electronic combined weight-height scale (Seca 769 Medical Scale; seca gmbh \& Co, Hamburg, Germany) and a measuring tape. The participants were weighed while they were lightly dressed and barefoot, and the weights were recorded to the closest $0.1 \mathrm{~kg}$. A participant's height was also measured to the closest $0.1 \mathrm{~cm}$ using an upright scale, which was behind the feet and shoulders while the participant was barefoot and looked straight ahead with her chin tucked in and the back of her head touched the wall. Besides, the waist circumference was measured at the midpoint between the lower margin of the last palpable rib and the top of the iliac crest while the participant was lightly dressed. In addition, the hip circumference was measured around the widest area of the buttocks $(26,27)$. The BMI was defined using the WHO criteria as a person's weight in kilograms divided by the square of their height in meters $\left(\mathrm{kg} / \mathrm{m}^{2}\right)$. If the participant's BMI was $18-25 \mathrm{~kg} / \mathrm{m}^{2}$, she was considered to have normal BMI Likewise, the woman was reported as being overweight or obese if her BMI was $25-30 \mathrm{~kg} /$ $\mathrm{m}^{2}$ or $\geq 30 \mathrm{~kg} / \mathrm{m}^{2}$, respectively. Furthermore, according to the WHO criteria, the following cases are considered as abnormal: (1) a waist circumference $>88 \mathrm{~cm}$; (2) a waist to hip ratio (WHR) $>0.85$; and (3) waist to height ratio $($ WHtR) $>0.5(27)$.

In the following section, the instruments applied in this study are described in detail.

\section{Questionnaire}

The questionnaire comprises 4 main parts. The first part gathers information about the participants': (1) sociodemographic variables including age, education, 
household income, marital status, and occupation; and (2) gynecological history such as the age of menarche, the menstrual cycle status, the age at menopause, if applicable, gravidity, parity, and the use of HRT.

Information regarding the individuals' lifestyle factors like their (1) smoking status and (2) exercise habits were collected in the second part of the questionnaire. Worth to mention that smoking status of the participants included both cigarettes and water pipe usage, and was calculated as the pack years for both using the Royal College of General Practitioners online calculator (28). The participants were categorized as follows: (I) inactive if they practiced no regular physical exercise; (II) mildly and physically active if they took exercise for $<90 \mathrm{~min} / \mathrm{wk}$; (III) moderately active if they did exercise from 90 to $<150 \mathrm{~min} / \mathrm{wk}$; and (IV) sufficiently active if they exercised for $\geq 150 \mathrm{~min} / \mathrm{wk}$.

In addition, the third part of the questionnaire included the Arabic version of the menopause rating scale (MRS), that is, it was translated into Arabic and validated by the investigators. The Arabic version of the MRS was reviewed by three professors and two assistant professors from the Department of Family and Community Medicine, the University of Jordan. All of the suggested modifications were discussed and applied in order to improve and make the items clearer. The MRS is a selfadministered questionnaire that has been validated and used in many clinical studies $(29,30)$, and its validity as a self-administered questionnaire has been emphasized as well (29). This questionnaire contains 11 items which are distributed into three main categories to be measured: Category (A), 'somatic and vasomotor symptoms' (4 items), namely, hot flashes and sweating, heart discomfort, sleep problems, and joint and muscular discomfort; Category B, 'urosexual symptoms' (3 items), that is, sexual problems, bladder problems, and vaginal dryness; and Category C, 'psychological symptoms' (4 items), namely, depressive moods, irritability, anxiety, and physical and mental exhaustion. Values 0 (no symptoms) to 4 (very severe symptoms) were allocated to each of these 11 items.

And finally, in the fourth part of the questionnaire which was developed by the investigators, the participants were questioned about 9 items associated with the quality of their lives. The items addressed the effects of menopausal symptoms on daily activities, shopping, using public transport, participating in social activities, particularly celebrations and meetings, and sexual relationships. Moreover, the impact of these symptoms on the participant's satisfaction with their overall appearance, weight, and eating habits was explored. Each of the items was scored on a scale ranging from 0 (no effect) to 4 (severely affected).

It is worth mentioning that the third and fourth parts of the questionnaire were reviewed for the validity of their content. Furthermore, before administering the questionnaire was pretested for language simplicity and clarity. Besides, the reliability of the questionnaire was tested in a pilot study of 40 patients (Cronbach $\alpha=$ 0.747). Well-trained research assistants were responsible for distributing the questionnaires, ensuring that all of the questions were answered, and completing the questionnaires for those patients who were unable to complete the forms independently because of literacy problems or poor eyesight.

\section{Statistical Analyses}

Statistical Package for the Social Sciences (SPSS) software, version 16 was used to analyze the data. In addition, multivariate logistic regression analysis was conducted to determine the studied risk factors that influenced the occurrence of different menopausal symptoms. The odds ratios and $95 \%$ CIs were calculated as well. A $P$ value $<$ 0.05 was considered statistically significance.

\section{Results}

The mean age of the studied population at menopause was 49.4 years. Table 1 presents the study cohort's sociodemographic data. Almost one-third of the study participants were $45-50$ years of age while $>66 \%$ of them were $<55$ years old. Besides, $14 \%$ of the participants were within the age range of 60-65 years. Most of the participants $(82.7 \%)$ were married whereas the remaining individuals were either widowed (11.1\%) or single (4.5\%). Regarding the participants' education, $<3 \%$ of them were illiterate while $13.4 \%$ of the participants had completed primary

Table 1. Sociodemographic Characteristics of the Samples

\begin{tabular}{|c|c|}
\hline Sociodemographic Variables & Number of Patients (\%) \\
\hline \multicolumn{2}{|l|}{ Age } \\
\hline $45-50$ & $117(32.6)$ \\
\hline $51-55$ & $123(34.3)$ \\
\hline $56-60$ & 68 (18.9) \\
\hline $61-65$ & $51(14.2)$ \\
\hline \multicolumn{2}{|l|}{ Marital Status } \\
\hline Married & $297(82.7)$ \\
\hline Widowed & $40(11.1)$ \\
\hline Divorced & $6(1.7)$ \\
\hline Single & $16(4.5)$ \\
\hline \multicolumn{2}{|l|}{ Educational level } \\
\hline Uneducated & $10(2.8)$ \\
\hline Primary school & $48(13.4)$ \\
\hline Secondary & $120(33.4)$ \\
\hline Diploma (2 years) & $102(28.4)$ \\
\hline Bachelor & $64(17.8)$ \\
\hline Post graduate (Master, PhD) & $15(4.2)$ \\
\hline \multicolumn{2}{|l|}{ Occupation } \\
\hline Housewife & $212(59.1)$ \\
\hline Retired & $98(27.3)$ \\
\hline Full time job & $49(13.6)$ \\
\hline Part time job & $0(0)$ \\
\hline \multicolumn{2}{|l|}{ Total monthly income (JOD) } \\
\hline$<800$ & $217(60.4)$ \\
\hline $800-1500$ & $131(36.5)$ \\
\hline$>1500$ & $11(3.1)$ \\
\hline
\end{tabular}


school education. And finally, $>50 \%$ of the participants had completed university degrees. Respecting the participants' occupations, housewives included more than $50 \%$ of the participants while the remaining were either retired (27\%) or full-time employed (13.6\%). In addition, with regard to the monthly income, $60 \%$ of the studied samples had a low monthly income of less than US $\$ 1128$ per month (800 JOD), and only $3 \%$ had an income of more than \$US2115 (1500 JOD). Table 2 shows the data on participants' exercise and smoking habits in addition to their obesity indices. As regards exercise, $22.6 \%$ of the participants exercised regularly for $\geq 150 \mathrm{~min} / \mathrm{wk}$ while $>38 \%$ of them did not exercise or exercised irregularly. Besides, with respect to the participants' smoking habits, $7 \%$ of them smoked cigarettes or used water pipes. Finally, regarding the obesity indices, $92.2 \%$ of the participants had BMIs $>25 \mathrm{~kg} / \mathrm{m}^{2}$ whereas the remaining participants had either abnormal WHtRs (81\%) or WHRs (39\%).

The results on the relationships between exercise, smoking, the obesity indices, and the MRS are presented in Table 3. The women who exercised irregularly or did not exercise were significantly more likely to experience irritability $(P=0.011)$, physical and mental exhaustion $(P=0.005)$, sexual problems $(P=0.007)$, and muscle and joint discomfort $(P=0.039)$ However, exercise was found to have a significant effect on other studied menopausal symptoms. The women whose smoking habits amounted to $\leq 20$ pack year were significantly more likely to experience bladder problems $(P=0.021)$, otherwise, no statistical significance was observed regarding the relationship between smoking and other menopausal

Table 2. Exercise Habits, Smoking and Obesity Indices in the Studied Population

\begin{tabular}{lc}
\hline & Number of Participants (\%) \\
\hline Exercise & $139(38.7)$ \\
No or Irregular & $78(21.7)$ \\
Less than $60 \mathrm{~min} / \mathrm{wk}$ & $61(17)$ \\
$60-90 \mathrm{~min} / \mathrm{wk}$ & $81(22.6)$ \\
More than $90 \mathrm{~min} / \mathrm{wk}$ & \\
Smoking & $333(92.8)$ \\
None & $19(5.3)$ \\
Cigarettes & $7(1.9)$ \\
Shisha & $1(0.3)$ \\
BMl & $27(7.5)$ \\
Underweight & $331(92.2)$ \\
Normal & \\
Overweight or obese & $68(18.9)$ \\
WHtR & $291(81.1)$ \\
Normal & \\
Abnormal & $218(60.7)$ \\
WHR & $141(39.3)$ \\
Normal &
\end{tabular}

Abbreviations: $\mathrm{BMI}$, body mass index; WHtR, waist to height ratio; WHR, waist to hip ratio. symptoms. Of the obesity indices, only the WHR had a significant association with the MRS, and the women with normal WHRs were at an increased risk of experiencing sexual problems $(P=0.042)$.

Table 4 summarizes the effects of exercise, smoking, and the obesity indices on the participants' menopausal symptoms and, hence, their effects on the women's quality of life. The presence of menopausal symptoms in participants who exercised irregularly or did not exercise negatively affected their domestic daily and shopping activities, and their sexual relationships as well. Moreover, those women who did not smoke were more likely to be unhappy about their weight. Nonetheless, there was no association between smoking and participant satisfaction in relation to weight. With the exception of the underweight women being significantly more likely to be unhappy about their weight $(P \leq 0.00)$, there were no correlations between any of the quality of life items studied and the BMI. Furthermore, women with abnormal WHtRs were significantly more likely to be unsatisfied about their appearance, in general, and their weight $(P \leq$ 0.00 ) while those with normal WHRs were significantly more likely to be unhappy about their sexual relationships. No relationship was found between any of the studied variables and using public transport, participation in social visits or family activities, and satisfaction about eating habits.

\section{Discussion}

The current study attempted to delve into the exercise habits and the prevalence of obesity among women in the menopausal age group and to determine whether exercise and obesity could affect the transition and the quality of life of the women through menopause. Based on the findings, exercise had a considerable influence on the quality of life of women at menopause. Although it was impossible to determine the effects of the different obesity indices on the quality of life, the importance of obesity as a stand-alone risk factor for other health-related issues should not be underestimated.

In the current study's cohort, $>92 \%$ of the participants were overweight or obese based on their BMIs. The findings of a study recently conducted in Jordan (8) showed that $53.1 \%$ of the women were obese (BMI $\geq 30 \mathrm{~kg} / \mathrm{m}^{2}$ ). In the present study, both overweight and obese women, that is, those with BMIs $>25 \mathrm{~kg} / \mathrm{m}^{2}$ were included in our cohort because both overweight and obesity variables are observed to be associated with a poor quality of life in menopausal women of different populations (31-34). This explains the difference in the rate of obesity between our cohort and that studied by Khader et al (8). The weight increase and higher incidence of obesity among menopausal women are considered as important public health problems (5). The findings of a study performed in Iran showed that women with high BMIs were at increased risk of experiencing vasomotor 


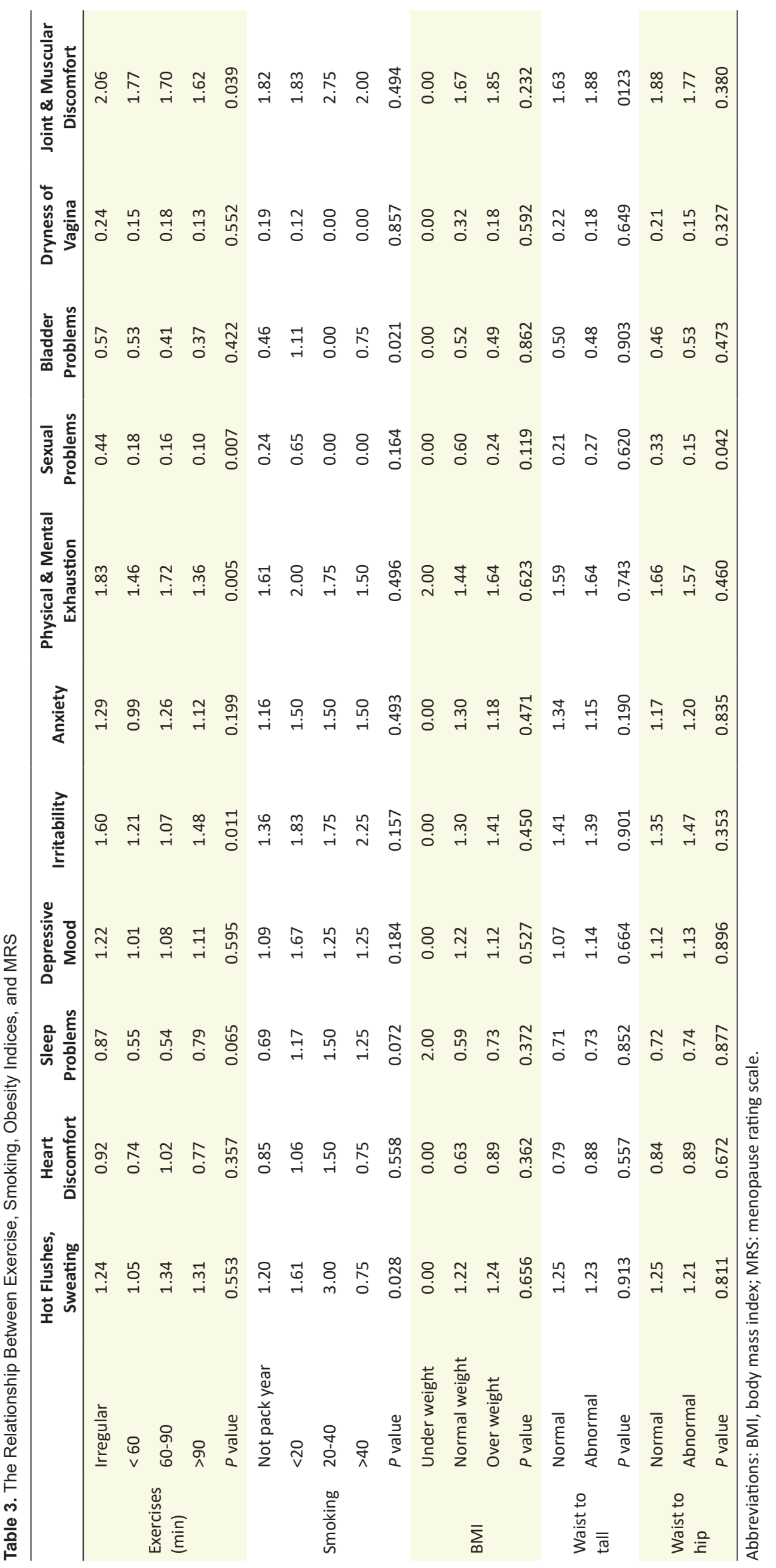




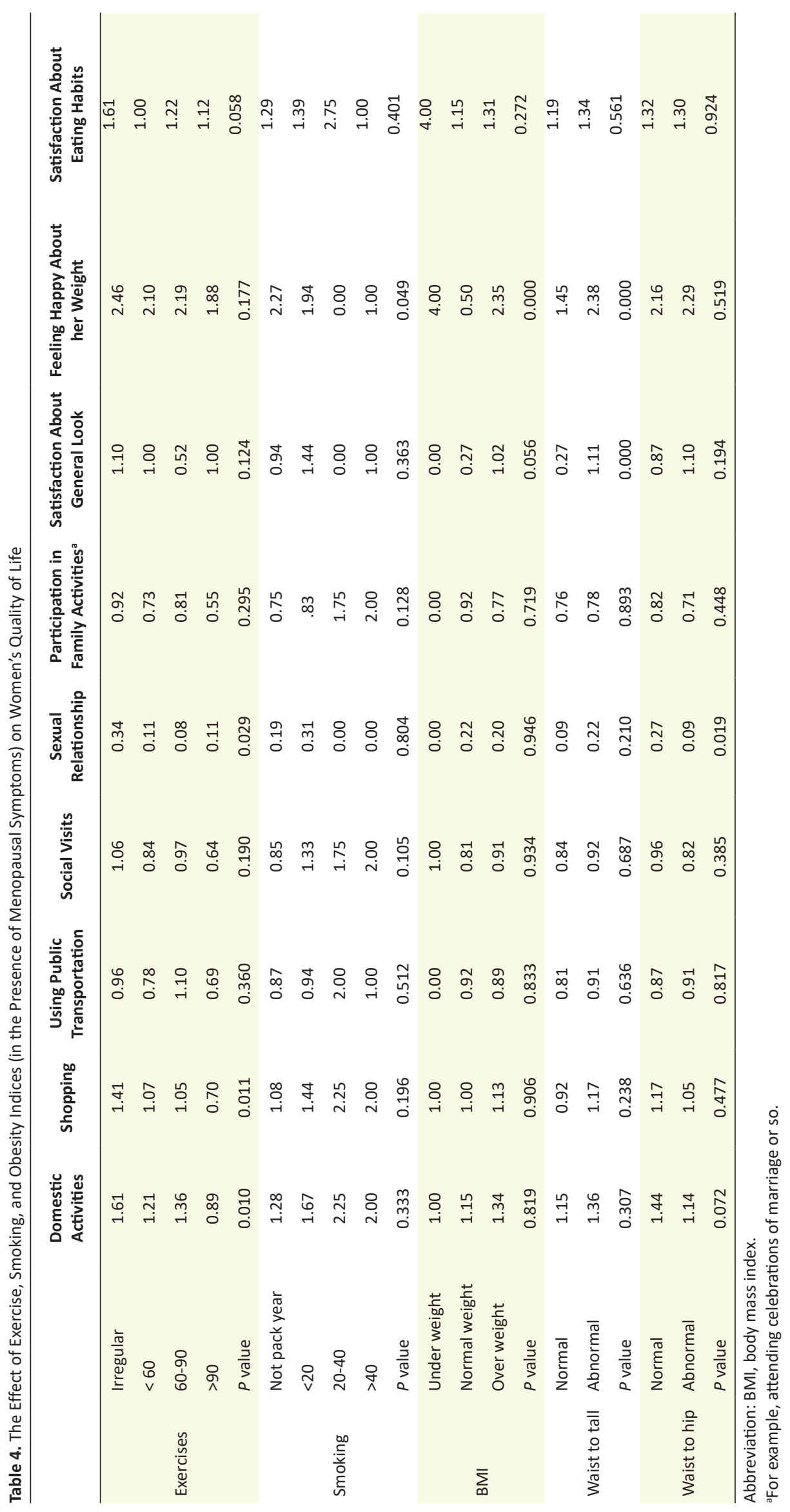


symptoms (35), which was an effect of obesity which was confirmed by the clinical research. In fact, significantly lower estradiol levels were found in premenopausal and overweight women compared with non-obese women (36). Furthermore, the menopausal transition impacted the distribution of body fat, and the increase in the level of visceral body fat was another important change that accompanied menopause (37). In this study, $81.1 \%$ of the participants had abnormal WHtRs and $39.3 \%$ of the participants had abnormal WHRs, both of which are important indicators of the distribution of body fat and abdominal obesity. Although no associations are evident between any of the obesity indices and menopausal symptoms' severity, obesity is a known risk factor for a long list of diseases including cerebrovascular diseases and many malignancies, especially as the women age.

Lack of exercise is an important cardiovascular risk factor. Within our cohort, $38 \%$ of the participants did not exercise or exercised irregularly, and $77 \%$ of them exercised ineffectively. The positive impact of exercise on the menopausal transition has been discussed in several publications. The findings of a study investigating the effects of exercise on overweight menopausal women over a year indicated that compared with the control subjects, the individuals who undertook regular aerobic exercise demonstrated significant increases in their hot flashes' severity and reductions in the risk of memory problems (18). In addition, the findings of several randomized controlled trials have shown that compared with women in non-exercise groups, those who enrolled to participate in an aerobic exercise showed improvements with respect to psychological wellbeing, mood, the health-related quality of life, and insomnia $(19,34,38)$. These findings are in line with the results of the present study that showed that women who did not exercise or exercised irregularly were more likely to experience mental and physical exhaustion and to experience more severe muscle pain and discomfort. Exercise has long been proven to be as effective as relaxation and meditation at managing patients with depression and anxiety disorders in the mildto-moderate stages (39), and the menopausal transition shares many of the mental and physical symptoms that are found in depression and anxiety disorders.

The findings of the current investigation regarding the effects of menopausal symptoms on women's quality of life revealed that these symptoms were more likely to affect women's daily activities, outdoor activities, and sexual relationships if they did not exercise or exercised irregularly. Besides, these findings suggest that exercise is not only important for alleviating some of the menopausal symptoms but also improves the quality of life of the women through the menopausal transition. Moreover, exercise induces weight loss and helps the women maintain a lower body weight, which are the ultimate goals for menopausal women.

\section{Conclusions}

In conclusion, physicians are recommended to be more aware of the high prevalence of obesity among older women. In addition, they are suggested to invest more time advising the patients about the important roles that weight and exercise play in disease prevention and improvement of women's quality of life throughout menopausal transition.

\section{Conflict of Interests}

Authors declare that they have no conflict of interests.

\section{Ethical Issues}

Informed consent was verbally obtained from each participant by well-trained research assistants who explained the purpose of the study. The Ethical Scientific Research Committee at the University of Jordan and the Institutional Review Board at the JUH approvals were obtained before commencing the study.

\section{Financial Support}

The research was funded by the Deanship of Academic Research at the University of Jordan.

\section{References}

1. Nowak NM. Development and evaluation of two mouse models for endometriosis focused on the involvement of the immune system in endometriosis establishment [dissertation]. Berlin: Chemie, Pharmazie der Freien Universität Berlin; 2008.

2. Grummer R. Animal models in endometriosis research. Hum Reprod Update. 2006;12(5):641-649. doi:10.1093/ humupd/dml026

3. Vernon MW, Wilson EA. Studies on the surgical induction of endometriosis in the rat. Fertil Steril. 1985;44(5):684694. doi:10.1016/S0015-0282(16)48988-0

4. Agarwal N, Subramanian A. Endometriosis - morphology, clinical presentations and molecular pathology. J Lab Physicians. 2010;2(1):1-9. doi:10.4103/0974-2727.66699

5. Benagiano G, Brosens I. History of adenomyosis. Best Pract Res Clin Obstet Gynaecol. 2006;20(4):449-463. doi:10.1016/j.bpobgyn.2006.01.007

6. Stratton P, Berkley KJ. Chronic pelvic pain and endometriosis: translational evidence of the relationship and implications. Hum Reprod Update. 2011;17(3):327346. doi:10.1093/humupd/dmq050

7. Beyene RT, Kavalukas SL, Barbul A. Intra-abdominal adhesions: Anatomy, physiology, pathophysiology, and treatment. Curr Probl Surg. 2015;52(7):271-319. doi:10.1067/j.cpsurg.2015.05.001

8. Klopfleisch R. Multiparametric and semiquantitative scoring systems for the evaluation of mouse model histopathology--a systematic review. BMC Vet Res. 2013;9:123. doi:10.1186/1746-6148-9-123

9. Barrier BF. Immunology of endometriosis. Clin Obstet Gynecol. 2010;53(2):397-402. doi:10.1097/ GRF.0b013e3181db7c33

10. Soares SR, Martinez-Varea A, Hidalgo-Mora JJ, Pellicer A. 
Pharmacologic therapies in endometriosis: a systematic review. Fertil Steril. 2012;98(3):529-555. doi:10.1016/j. fertnstert.2012.07.1120

11. Paul Dmowski W, Braun DP. Immunology of endometriosis. Best Pract Res Clin Obstet Gynaecol. 2004;18(2):245-263. doi:10.1016/j.bpobgyn.2004.02.001

12. Giudice LC. Clinical practice. Endometriosis. N Engl J Med. 2010;362(25):2389-2398. doi:10.1056/NEJMcp1000274

13. Sampson JA. Heterotopic or misplaced endometrial tissue. Am J Obstet Gynecol. 1925;10(5):649-664. doi:10.1016/ S0002-9378(25)90629-1

14. Leyland N, Casper R, Laberge P, Singh SS. Endometriosis: diagnosis and management. J Obstet Gynaecol Can. 2010;32(7 Suppl 2):S1-32. doi:10.1016/S17012163(16)34589-3

15. Paula R Jr, Oliani AH, Vaz-Oliani DC, D’Avila SC, Oliani $\mathrm{SM}$, Gil CD. The intricate role of mast cell proteases and the annexin A1-FPR1 system in abdominal wall endometriosis. J Mol Histol. 2015;46(1):33-43. doi:10.1007/s10735-0149595-y

16. Machado DE, Berardo PT, Palmero CY, Nasciutti LE. Higher expression of vascular endothelial growth factor (VEGF) and its receptor VEGFR-2 (Flk-1) and metalloproteinase-9 (MMP-9) in a rat model of peritoneal endometriosis is similar to cancer diseases. J Exp Clin Cancer Res. 2010;29:4. doi:10.1186/1756-9966-29-4

17. Woodward PJ, Sohaey R, Mezzetti TP Jr. Endometriosis: radiologic-pathologic correlation. Radiographics. 2001;21(1):193-216; questionnaire 288-194. doi:10.1148/ radiographics.21.1.g01ja14193

18. Sutrisno S, Aprina H, Simanungkalit HM, et al. Genistein modulates the estrogen receptor and suppresses angiogenesis and inflammation in the murine model of peritoneal endometriosis. J Tradit Complement Med. 2018;8(2):278-281. doi:10.1016/j.jtcme.2017.03.002

19. Hirata T, Osuga Y, Yoshino O, et al. Development of an experimental model of endometriosis using mice that ubiquitously express green fluorescent protein. Hum Reprod. 2005;20(8):2092-2096. doi:10.1093/humrep/ dei012

20. Greaves E, Cousins FL, Murray A, et al. A novel mouse model of endometriosis mimics human phenotype and reveals insights into the inflammatory contribution of shed endometrium. Am J Pathol. 2014;184(7):1930-1939. doi:10.1016/j.ajpath.2014.03.011

21. Grummer R. Animal models in endometriosis research. Hum Reprod Update. 2006;12(5):641-649. doi:10.1093/ humupd/dml026

(c) 2019 The Author (s); This is an open-access article distributed under the terms of the Creative Commons Attribution License (http://creativecommons.org/licenses/by/4.0), which permits unrestricted use, distribution, and reproduction in any medium, provided the original work is properly cited. 